\title{
A Unique Flavin Mononucleotide-Linked Primary Alcohol Oxidase for Glycopeptide A40926 Maturation
}

\author{
Yi-Shan Li, ${ }^{\dagger, \S}$ Jin-Yuan Ho, ${ }^{\dagger, l}$ Chia-Chi Huang, ${ }^{\dagger, \|}$ Syue-Yi Lyu, ${ }^{\dagger, \S}$ Chun-Yen Lee, ${ }^{\dagger, \S}$ Yu-Ting Huang, ${ }^{\dagger, \S}$ \\ Chang-Jer Wu,, Hsiu-Chien Chan, ${ }^{\dagger, \perp}$ Chuan-Jiuan Huang,,,$\S$ Ning-Shian Hsu, ${ }^{\dagger, \S}$ \\ Ming-Daw Tsai,, ,‡ and Tsung-Lin $\mathrm{Li}^{\star}, \dagger$ \\ Genomics Research Center and Institute of Biological Chemistry, Academia Sinica, Taipei 115, Department of Food \\ Science, National Taiwan Ocean University, Keelung 202, Institute of Biochemical Science, National Taiwan \\ University, Taipei 106, and Institute of Bioinformatics and Structure Biology, National Tsing Hua University, \\ Hsinchu 300, Taiwan, Republic of China
}

Received August 1, 2007; E-mail: tlli@gate.sinica.edu.tw

The teicoplanin homologue A40926 differs from teicoplanin (1) mainly in lacking the GlcNAc on residue 6 (r6) and the replacement of the $\mathrm{N}$-acyl glucosaminyl C-6 $\mathrm{OH}$ substituent on residue 4 (r4) with a $\mathrm{COOH}$ group. ${ }^{1} \mathrm{We}$ and others have reported that three enzymes, Dbv9, Dbv21, and Dbv8, act sequentially to add the $N$-acyl glucosaminyl moiety to 4 of teicoplanin or A40926 aglycone (2). ${ }^{2}$ The $N$-acyl glucosaminyl substituent might also undergo further C-6 oxidation to $N$-acyl aminoglucuronic acid during A40926 biosynthesis, but whether this occurs as the last step in this biosynthesis series has yet to be determined. Herein we report that Dbv29, encoded by a gene in the A40926 biosynthetic gene cluster, is an flavin mononucleotide (FMN)-dependent primary alcohol glycopeptide hexose oxidase which carries out a 4-electron oxidation reaction to complete $N$-acyl aminoglucuronic acid biosynthesis for A40926 maturation. To the best of our knowledge, this is the first biochemically characterized hexose oxidase of this kind. Previously a hexose oxidase is assumed to be an flavin adenine dinucleotide (FAD)-dependent hexose oxidase that oxidizes the C-1 hydroxyl group of $\beta$-D-glucose to D-glucono-1,5-lactone with concomitant production of $\mathrm{H}_{2} \mathrm{O}_{2}$. The $\mathrm{C}-6$ hydroxyl group of uridine 5'-diphosphate (UDP)-glucose or guanosine $5^{\prime}$-diphosphate (GDP)-mannose is oxidized to UDP-glucuronic acid or GDP-mannuronic acid, respectively, by nicotinamide adenine dinucleotide (phosphate) $\left(\mathrm{NAD}^{+} / \mathrm{NADP}^{+}\right)$-dependent dehydrogenases via two oxidation reactions, in which a substrate-enzyme intermediate is formed through a cysteine thioester linkage in the active site. ${ }^{3}$ However, galactose oxidase oxidizes the C-6 hydroxyl group of galactose to the corresponding aldehyde through a radical-coupled copper oxidation reaction. ${ }^{3}$

The $d b v 29$ gene has been annotated as a putative oxidoreductase ${ }^{2}$ and its gene product may be involved in the biosynthesis of $\mathrm{N}$-acyl aminoglucuronic acid. To test this assumption, recombinant $N$ terminal His ${ }_{6}$-tagged Dbv29 was cloned, expressed in E. coli, and purified to homogeneity (Supporting Information, SI Figure 2). The purified protein solution was bright yellow (absorbance maxima at 330 and $430 \mathrm{~nm}$ ), suggesting that it may contain a flavinlike cofactor. $^{4}$ However, the cofactor was not released from the denatured holoenzyme by heating or treatment with acids or organic solvents. Recently, the structure of the oxidoreductase AknOx, which catalyzes the conversion of rhodinose to L-aculose (secondary alcohol to $\alpha-\beta$ unsaturated ketone) in the biosynthesis of the polyketide antibiotic aclacinomycin, was solved and the FAD

† Genomics Research Center, Academia Sinica.

¥ Institute of Biological Chemistry, Academia Sinica.

$\S$ National Taiwan Ocean University.

"National Taiwan University

${ }^{\perp}$ National Tsing Hua University.

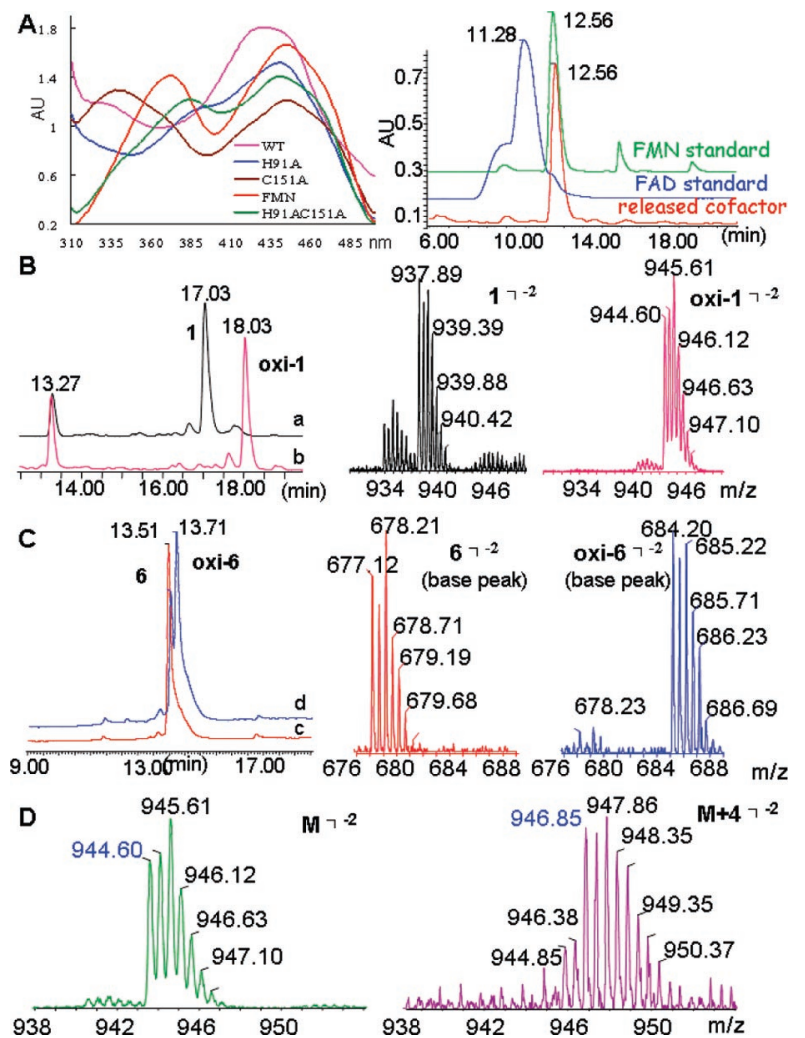

Figure 1. UV, LC, and mass spectra data: (A) demonstration of covalent linkage (left) and FMN cofactor (right); (B) incubation of compound $\mathbf{1}$ in the absence (a) or presence (b) of Dbv29; (C) incubation of compound 6 in the absence (c) or presence (d) of Dbv29; (D) mass spectra in the presence of $\mathrm{D}_{2} \mathrm{O}$ or ${ }^{18} \mathrm{O}_{2}$ or $\mathrm{H}_{2}{ }^{18} \mathrm{O}_{2}$ (left) and $\mathrm{H}_{2}{ }^{18} \mathrm{O}$ (right) (mass spectra shown with doubly charged negative ions).

cofactor was shown to be covalently linked to residues His70 and Cys130.5 Sequence alignment of AknOx and Dbv29 (SI) showed that these residues are conserved in Dbv29 (H91 and C151). When H91 or C151 of Dbv29 was mutated to alanine, the enzyme solution remained yellow (although the absorbance peak at $330 \mathrm{~nm}$ was red-shifted; Figure 1A, left panel) and the colored substance was not released by denaturation. The double mutant also retained the yellow color, but heat or acid treatment resulted in release of the colored material. When the enzyme-free solution was subjected to HPLC/MS analysis, the colored agent unexpectedly turned out to be FMN (Figure 1A, right panel). Thus, the cofactors in the two enzymes are covalently bound to their respective enzyme in a similar manner, but Dbv29 uses FMN rather than FAD. 


\section{Scheme 1}

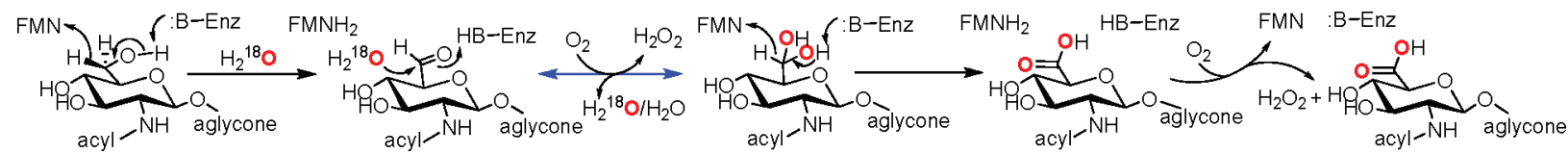

Scheme 2

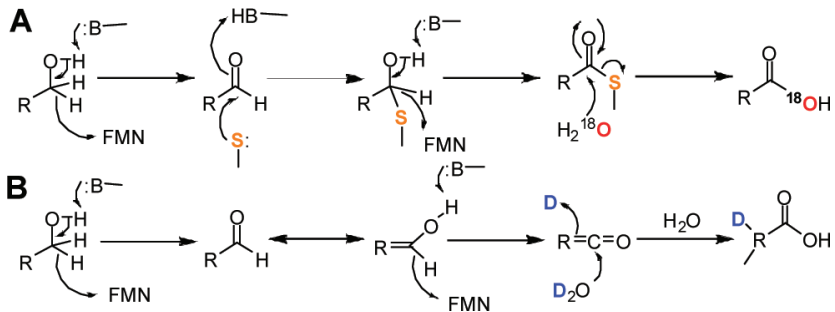

Table 1. Reaction Activity (Rx) of Dbv29 on Different Substrates ${ }^{a}$

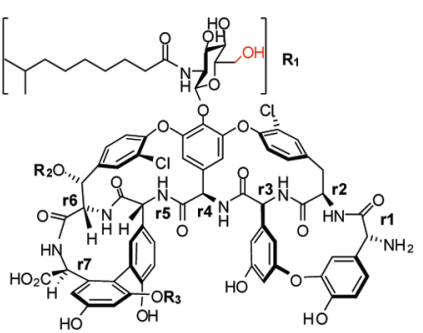

\begin{tabular}{c|cccl} 
& $\mathbf{R}_{\mathbf{1}}$ & $\mathbf{R}_{\mathbf{2}}$ & $\mathbf{R}_{\mathbf{3}}$ & $\mathbf{R x}$ \\
\hline $\mathbf{1}$ & GlcNAcyl & GlcNAc & Man & + \\
$\mathbf{2}$ & H & H & H & - \\
$\mathbf{3}$ & GlcN & GlcNAc & Man & - \\
$\mathbf{4}$ & GlcN & GlcNAc & H & - \\
$\mathbf{5}$ & GlcN & H & H & - \\
$\mathbf{6}$ & GlcNAc & H & H & + \\
$\mathbf{7}$ & H & GlcNAc & H & -
\end{tabular}

${ }^{a}$ The compounds that can serve as a substrate of Dbv29 are marked $(+)$, in which the $\mathrm{OH}$ substituent highlighted in red is converted into $\mathrm{COOH}$.

To test the activity of Dbv29 on different substrates (Table 1), the reaction solution (50 mM HEPES, $\mathrm{pH}$ 7.4) containing 1 was incubated with the purified protein at $25{ }^{\circ} \mathrm{C}$ for $4 \mathrm{~h}$, then the enzyme-free reaction solution was subjected to HPLC/MS analysis. The LC trace revealed a new peak at $18.0 \mathrm{~min}$ (Figure 1B, left panel) that was shown to have higher mw by 14 (Figure 1B, right panels), supporting oxidation of $\mathrm{CH}_{2} \mathrm{OH}$ to $\mathrm{COOH}$. MS/MS analysis confirmed that the oxidation occurred on the C-6 $\mathrm{N}$-acyl glucosaminyl moiety (SI Figure 9). Dbv29 was also tested for activity on UDP- $N$-Ac-glucosamine/UDP-glucosamine, but no new product was seen (SI), suggesting that oxidation does not occur at the stage of dinucleotide sugars. Dbv29 was also tested using compounds 3-5, but no new products were seen (SI). When tested on compound 6, a new peak was seen at $13.7 \mathrm{~min}$, which was confirmed to be the corresponding oxidized compound $\mathbf{6}$ (Figure 1C). The oxidized compound $\mathbf{6}$ was not deacetylated by Dbv21 (SI), suggesting that the introduced carbonyl group abolished the deacetylation activity of Dbv21. ${ }^{2}$ Taken together, we conclude that the C-6 hydroxy oxidation should occur no earlier than the reacylation of the glucosaminyl pseudoaglycone with a long aliphatic side chain $^{2}$ and that the carbonyl group of the acyl side chain may be involved in substrate recognition regardless of the carbon chain length. In addition, compound $\mathbf{7}$ failed to produce a product (SI), suggesting that Dbv29 works strictly on the $N$-acyl glucosaminyl substituent of residue 4 of the pseudoaglycone and is unaffected by any other sugar(s) attached to residue 6 and/or 7 (Table 1).

Since $\mathrm{H}_{2} \mathrm{O}_{2}$ production was detected in a peroxidase-coupled assay and consumption of $\mathrm{O}_{2}$ over the reaction time course was recorded with an oxygen electrode (SI), Dbv29 was concluded to be an oxidase. Since it is most reasonable that the same mechanism is used to convert $\mathrm{FMNH}_{2}$ back to FMN for the two oxidation steps, and since the product produced in $\mathrm{H}_{2}{ }^{18} \mathrm{O}$ buffer showed dominant $\mathrm{M}+4$ species (Figure 1D), we propose a working mechanistic model in Scheme 1, where water attacks an aldehyde intermediate to generate a diol species that would allow exchange of both oxygens with ${ }^{18} \mathrm{O}$ and the second oxidation to occur. Here we report additional results that rule out alternative mechanisms. (i) In Scheme 2A, Dbv29 acts like dinucleotide-sugar dehydrogenases, in which a thiol-containing residue is required to form a tetrahedral intermediate in the catalytic center. ${ }^{3}$ However, when all four Cys residues in Dbv29 were separately mutated to Ala, no effect was seen on the activity (SI Figure 21). (ii) Scheme 2B involves an enol/ keto tautomerization mechanism for two consecutive two-electron oxidations. If the reaction is carried out in the presence of $\mathrm{D}_{2} \mathrm{O}$, a deuterium atom would be found in the final product (route $\mathrm{B}$ ); however, this did not occur (SI Figure 19). (iii) Peroxide or $\mathrm{H}_{2}{ }^{18} \mathrm{O}_{2}$ produced in the first step might act as a direct oxidant for the second oxidation. If either ${ }^{18} \mathrm{O}_{2}$ or $\mathrm{H}_{2}{ }^{18} \mathrm{O}_{2}$ served as the oxidant in the oxidation, an ${ }^{18} \mathrm{O}$ atom(s) should be seen in the final product; however, this was not the case under the above conditions (SI Figure 18).

Further experiments are in progress to refine the working model. Metal ions are probably not required to enhance the nucleophilicity of the water, as the activity was unchanged in the presence of 25 mM EDTA (SI Figure 20). It was possible that tyrosine residues Y165 and Y473 in Dbv29, which correspond to the proposed general base pairs in AknOx, might coordinate to deprotonate the C-6 hydroxyl group, triggering hydride transfer. ${ }^{5}$ However, the activities of the two mutants Y165F and Y473F and of Y370F and Y470F (mutants of other nearby candidates) were unchanged. Which base(s) are involved should be determined from the structure of Dbv29 complexed with substrates or analogues. In addition, single mutant (H91A or C151A) showed comparable activity to wild type, while the double mutant retained only $10 \%$ activity, suggesting that FMN needs properly placed in the active site. In summary, we have shown that Dbv29 is a new class of hexose oxidase $\left(K_{\mathrm{m} \text { teicoplanin }}\right.$ of $531 \mu \mathrm{M}$ and $k_{\text {cat teicoplanin }}$ of $\left.4.08 \times 10^{2} \mathrm{~s}^{-1}\right)$ that completes $N$-acyl aminoglucuronic acid biosynthesis. The characterized enzyme might provide a new way for biochemically transforming currently used glycopeptide drugs. Detailed functionmechanism analyses of the enzyme will expand our knowledge of a new class of enzyme, the glycopeptide hexose oxidases.

Acknowledgment. TLL thanks the National Science Council for financial support (Grant NSC 95-2311-B001-076).

Supporting Information Available: Experimental procedures, chromatograms, spectra, and sequence alignments. This material is available free of charge via the Internet at http://pubs.acs.org.

\section{References}

(1) Sosio, M.; Donadio, S. J. Ind. Microbiol. Biotechnol. 2006, 33, 69-76.

(2) (a) Sosio, M.; Stinchi, S.; Beltrametti, F.; Lazzarini, A.; Donadio, S. Chem Biol. 2003, 10, 541-549. (b) Ho, J. Y.; Huang, Y. T.; Wu, C. J.; Li, Y. S.; Tsai, M. D.; Li, T. L. J. Am. Chem. Soc. 2006, 128, 13694-13695. (c) Truman, A. W.; Robinson, L.; Spencer, J. B. Chembiochem 2006, 7 1670-1675. (d) Kruger, R. G.; Lu, W.; Oberthür, M.; Tao, J.; Kahne, D.; Walsh, C. T. Chem. Biol. 2005, 12, 131-140.

(3) (a) Leskovac, V.; Trivic, S.; Wohlfahrt, G.; Kandrac, J.; Pericin, D. Int J. Biochem. Cell Biol. 2005, 37, 731-750. (b) Whittaker, J. M. Chem. Rev. 2003, 103, 2347-2363. (c) Campbell, R. E.; Mosimann, S. C.; van De Rijn, I.; Tanner, M. E.; Strynadka, N. C. Biochemistry 2000, 39, 70127023. (d) Snook, C. F.; Tipton, P. A.; Beamer, L. J. Biochemistry 2003, 42, 4658-4668.

(4) Karplus, P. A.; Fox, K. M.; Massey, V. FASEB J. 1995, 9, 1518-1526.

(5) Alexeev, I.; Sultana, A.; Mantsala, P.; Niemi, J.; Schneider, G. Proc. Natl. Acad. Sci. U.S.A. 2007, 104, 6170-6175.

JA075748X 